\title{
BROWN-COMENETZ DUALITY AND THE ADAMS SPECTRAL SEQUENCE
}

\author{
MARK MAHOWALD AND CHARLES REZK
}

\begin{abstract}
We show that the class of $p$-complete connective spectra with finitely presented cohomology over the Steenrod algebra admits a duality theory related to Brown-Comenetz duality. This construction also produces a full-plane version of the classical Adams spectral sequence for such spectra, which converges to the homotopy groups of a "finite" localization.
\end{abstract}

\section{INTRODUCTION}

In the paper [3], Brown and Comenetz introduced a notion of duality into stable homotopy. In [4] Hopkins and Gross showed that this notion in certain situations is closely connected with Spanier-Whitehead duality. In this note we wish to explore this connection and investigate it in connection with Adams spectral sequence considerations. In particular, we study a class of spectra which we call $f p$-spectra (Section 3). These are connective, $p$-complete spectra whose mod $p$ cohomology is finitely presented over the Steenrod algebra; that is, the cohomology of such a spectrum is described by a finite set of generators together with a finite set of relations. This class of spectra includes the Johnson-Wilson spectra $B P\langle n\rangle$, connective $K$-theories, and the "higher" connective $K$-theory spectrum $e o_{2}$. The class of fpspectra also includes some objects whose Bousfield $L_{n}$-localizations are the same as $L_{n}$ localizations of finite complexes, at least in some cases. A classical example is the connective image-of- $J$ spectrum, whose $L_{1}$-localization is $L_{1} S^{0}$. It follows from calculations of Shimomura and Yabe that a -1-connective cover of $L_{2} S^{0}$ at primes $p \geq 5$ is also an fp-spectrum (Proposition 3.7).

We show that the category of fp-spectra admits a notion of duality (Theorem 8.11). This duality is related to both Brown-Comenetz duality and SpanierWhitehead duality. The dual $W X$ of an fp-spectrum $X$ will be defined to be the Brown-Comenetz dual of the fiber of the map $X \rightarrow L_{n}^{f} X$ to the "finite localization" of $X$ (for $n$ sufficiently large). The dual $W X$ is itself an fp-spectrum. This duality is related to Spanier-Whitehead duality through its action on cohomology, in the following sense. If $H^{*} X \approx A^{*} \otimes_{A^{*}(n)} M$ where $A^{*}(n) \subset A^{*}$ is a finite sub-Hopf algebra of the Steenrod algebra, and $M$ is a finite $A^{*}(n)$ module, then $H^{*} W X \approx A^{*} \otimes_{A^{*}(n)} \check{M}$, where $\check{M} \approx \operatorname{hom}_{\mathbb{F}_{p}}\left(M, \mathbb{F}_{p}\right)$ is the "Spanier-Whitehead dual" of $M$ as a finite module over $A^{*}(n)$.

Because of this duality, the $L_{n}^{f}$-localization of an fp-spectrum is quite computable. We show that there is a full-plane spectral sequence computing $\pi_{*} L_{n}^{f} X$, with $E_{2}$-term a "Tate cohomology" of $H^{*} X$ as a module over the Steenrod algebra

Date: September 8, 1998.

Copyright (C) 1998 The Johns Hopkins University Press. This article first appeared in the American Journal of Mathematics, Volume 00, Issue 00, Month, Year, pages 000-000. 
(Proposition 6.3 and Theorem 7.1). In all cases we know of $L_{n}^{f} X \approx L_{n} X$ for an fp-spectrum $X$.

1.1. Organization of the paper. In Section 2 we discuss modules and comodules which are finitely presented over the Steenrod algebra. In Section 3 we define the notion of fp-spectra, and give examples. In Section 4 we discuss a duality functor for finitely presented comodules over the Steenrod algebra, which is related to the action of Brown-Comenetz duality on Eilenberg-Mac Lane spectra discussed in Section 5. In Section 6 we note that an fp-spectrum admits a tower associated to a spectral sequence whose $E_{2}$-term is the "Tate cohomology" of the homology of the spectrum, and in Section 7 show that such a tower realizes the localization functor $L_{n}^{f}$. In Section 8 we describe the duality theory of fp-spectra. In Section 9 we calculate some examples.

1.2. Notation. In this paper we work at one prime $p$ at a time. We let $A^{*}$ denote the $\bmod p$ Steenrod algebra, and $A_{*}$ denote the dual mod $p$ Steenrod algebra.

Unless otherwise indicated, all vector spaces, modules, and comodules in this paper are graded. If $V$ is a graded vector space over $\mathbb{F}_{p}$, then $\check{V}$ denotes the linear dual $\operatorname{hom}\left(V, \mathbb{F}_{p}\right)$. If $V$ is a left comodule over a graded Hopf algebra $B$, then $\check{V}$ is taken to be a left comodule over $B$, via the canonical anti-automorphism $\chi$ of $B$.

When dealing with graded objects, we use the following sign convention: a sign is introduced whenever two symbols of odd degree are commuted.

\section{Finitely presented modules and Comodules over the Steenrod ALGEBRA}

A module $M$ over the mod $p$ Steenrod algebra $A^{*}$ is called finitely presented if it fits in an exact sequence of modules

$$
A^{*} \otimes V_{1} \rightarrow A^{*} \otimes V_{0} \rightarrow M \rightarrow 0
$$

where $V_{i}$ for $i=0,1$ are finite dimensional graded $\mathbb{F}_{p}$-vector spaces. Likewise, a comodule $N$ over the dual mod $p$ Steenrod algebra $A_{*}$ is called finitely presented if it fits in an exact sequence of comodules

$$
0 \rightarrow N \rightarrow A_{*} \otimes V_{0} \rightarrow A_{*} \otimes V_{1}
$$

where $V_{i}$ for $i=0,1$ are finite dimensional graded $\mathbb{F}_{p}$-vector spaces. Because all finitely presented modules and comodules are of finite type, we can pass easily between comodule and module language by taking vector space duals.

The Steenrod algebra $A^{*}$ is a union of finite-dimensional sub-Hopf algebras. For example, $A^{*}=\bigcup_{n} A^{*}(n)$, where $A^{*}(n) \subset A^{*}$ is a finite dimensional sub-Hopf algebra of the Steenrod algebra, generated as an algebra by $\left\{S q^{2^{i}} \mid i \leq n+1\right\}$ if $p=2$ and by $\left\{\beta, P^{p^{i}} \mid i \leq n\right\}$ if $p$ is odd. Recall that $A^{*}$ is free as an $A^{*}(n)$-module.

\section{Lemma 2.1.}

1. A module $M$ over $A^{*}$ is finitely presented if and only if it is of the form $M \approx A^{*} \otimes_{E} N$ for some finite dimensional sub-Hopf algebra $E \subset A^{*}$ and some finite dimensional $E$-module $N$.

2. Every map $f: M \rightarrow M^{\prime}$ of finitely presented A-modules is of the form $f \approx$ $A^{*} \otimes_{E} g: A^{*} \otimes_{E} N \rightarrow A^{*} \otimes_{E} N^{\prime}$ for some finite dimensional sub-Hopf algebra $E \subset A^{*}$ and some map $g: N \rightarrow N^{\prime}$ of finite dimensional E-modules. 
BROWN-COMENETZ DUALITY AND THE ADAMS SPECTRAL SEQUENCE

Proof. Any finite sub-Hopf algebra $E \subset A^{*}$ is contained in $A^{*}(n)$ for some $n \geq 0$, whence part 1 is [12, Ch. 13, Prop. 2(a)]. Part 2 follows by similar arguments.

\section{Proposition 2.2.}

1. The kernel and cokernel of a map of finitely presented $A^{*}$-modules are finitely presented.

2. A retract of a finitely presented $A^{*}$-module is finitely presented.

3. If $0 \rightarrow M^{\prime} \rightarrow M \rightarrow M^{\prime \prime} \rightarrow 0$ is a short exact sequence in which $M^{\prime}$ and $M^{\prime \prime}$ are finitely presented, then $M$ is also finitely presented.

Proof. Since $A^{*}$ is $A^{*}(n)$-free, the functor $A^{*} \otimes_{A^{*}(n)}(-)$ is exact, and hence part 1 follows from (2.1).

To prove part 2, note that a retract $N$ of $M$ is the kernel of an idempotent self-map $e: M \rightarrow M$. Hence part 2 follows from part 1 .

The proof of part 3 is a standard result about finitely presented modules over any ring.

Proposition 2.3. Suppose $M$ is an $A^{*}$-module and $F$ is a finite $A^{*}$-module. Then $M$ is finitely presented if and only if $M \otimes F$ is.

Proof. It is immediate from (2.2) that $M \otimes F$ is finitely presented if $M$ is, since $F$ admits a finite filtration whose subquotients are copies of $\mathbb{F}_{p}$.

Suppose $M \otimes F$ is finitely presented. Since $F$ is a finite module, we can choose a "pinch" map $\pi: F \rightarrow \Sigma^{d} \mathbb{F}_{p}$ to a "bottom-dimensional cell" of $F$, and we can write $i: \bar{F} \rightarrow F$ for the kernel of $\pi$. Then there is an exact sequence

$$
M \otimes \bar{F} \otimes F \stackrel{1 \otimes i \otimes \pi}{\longrightarrow} M \otimes F \stackrel{1 \otimes \pi}{\longrightarrow} M \rightarrow 0
$$

which exhibits $M$ as a cokernel of a map between finitely presented modules, and the result follows from $(2.2)$.

Remark 2.4. Note that (2.1), (2.2), and (2.3) dualize to similar statements about finitely presented comodules. We will not state the dual form of these results, although we will make use of them in what follows.

2.5. Homological algebra for finitely presented comodules. Henceforth we concentrate on finitely presented comodules. We let $\mathcal{M}_{\mathrm{fp}}$ denote the category of finitely presented comodules over $A_{*}$. By $(2.2)$ we see that $\mathcal{M}_{\text {fp }}$ is an abelian category.

Proposition 2.6. The dual Steenrod algebra $A_{*}$, viewed as a $A_{*}$-comodule, is both projective and injective in $\mathcal{M}_{\mathrm{fp}}$, and $\mathcal{M}_{\mathrm{fp}}$ has enough projectives and injectives.

Proof. It is clear that $A_{*}$ is injective in the full category of $A_{*}$ comodules, and hence $A_{*}$ is injective in $\mathcal{M}_{\mathrm{fp}}$ and there are enough injectives in $\mathcal{M}_{\mathrm{fp}}$. To prove that $A_{*}$ is projective, consider a surjection $M \rightarrow M^{\prime}$ of finitely presented comodules. By (2.1), this map is extended up from a surjection $N \rightarrow N^{\prime}$ of finite $A_{*}(n)$-comodules. Since $\operatorname{hom}_{A_{*}}\left(A_{*}, M\right) \approx \operatorname{hom}_{A_{*}(n)}\left(A_{*}, N\right)$ and $A_{*}$ is $A_{*}(n)$-free, any map $A_{*} \rightarrow M^{\prime}$ can be lifted to a map to $M$. Furthermore, we can always produce enough maps from $A_{*}$ to a finitely presented comodule, and thus $\mathcal{M}_{\mathrm{fp}}$ has enough projectives.

Remark 2.7. It is known that the Steenrod algebra is injective as an $A^{*}$-module over itself $\left[12\right.$, p. 201]. It would be interesting to know whether $A_{*}$ is projective as a comodule over itself, without the restriction to the finitely presented category. 
Given a finitely presented comodule $M$, one can define its Tate cohomology as follows. By (2.6) we can choose injective and projective resolutions

$$
0 \rightarrow M \rightarrow C_{0} \rightarrow C_{1} \rightarrow C_{2} \rightarrow \ldots
$$

and

$$
\ldots \rightarrow C_{-3} \rightarrow C_{-2} \rightarrow C_{-1} \rightarrow M \rightarrow 0
$$

by finitely generated free $A_{*}$-comodules. By gluing the ends together, we obtain an unbounded complex

$$
\ldots \rightarrow C_{-2} \rightarrow C_{-1} \rightarrow C_{0} \rightarrow C_{1} \rightarrow \ldots
$$

which in each degree is injective in the category of comodules. For $s \in \mathbb{Z}$ define

$$
H_{\text {Tate }}^{s}(M)=H^{s}\left[\operatorname{hom}_{A_{*}}\left(\mathbb{F}_{p}, C_{\bullet}\right)\right] .
$$

Of course, if we can write $M \approx A_{*} \otimes_{A_{*}(n)} N$ for some $A_{*}(n)$-comodule $N$, and we choose resolutions $0 \rightarrow N \rightarrow D_{\bullet}$ and $D_{-\bullet} \rightarrow N \rightarrow 0$ of $N$ by finite free $A_{*}(n)$ comodules, then we see that

$$
H_{\text {Tate }}^{s}(M) \approx H^{s}\left[\operatorname{hom}_{A_{*}(n)}\left(\mathbb{F}_{p}, D_{\bullet}\right)\right] .
$$

These groups are the same as morphisms in the stable category of $A_{*}(n)$-modules, as is shown in [7, Sec. 9.6].

\section{FP-SPECTRA}

In this section we define the notion of fp-spectra, and produce several examples. Recall that we work in the category of $p$-local spectra.

We first note the following theorem of Mitchell.

Theorem 3.1 (Mitchell). [13] For each $n$ there exists a non-trivial finite complex $F$ such that $H_{*} F$ is $A_{*}(n)$-free.

If $X$ is a spectrum, say that $\pi_{*} X$ is finite if $\pi_{k} X=0$ for all but finitely many $k \in \mathbb{Z}$, and is a finite group otherwise.

Proposition 3.2. Suppose $X$ is a connective, $p$-complete spectrum. Then the following are equivalent.

1. $H_{*} X$ is finitely presented as a comodule over the Steenrod algebra.

2. $H_{*} X \approx A_{*} \otimes_{A_{*}(n)} M$ for some $n \geq 0$ and some finite $A_{*}(n)$-comodule $M$.

3. There exists a non-trivial finite complex $F$ such that $X \wedge F$ is a finite wedge of suspensions of mod p Eilenberg-Mac Lane spectra.

4. There exists a non-trivial finite complex $F$ such that $\pi_{*}(X \wedge F)$ is finite.

Proof. The equivalence of 1 and 2 is just (2.1). Likewise, 4 is immediate given 3 .

To show that 2 implies 3 , we let $F$ be as in (3.1), with $H_{*} F$ free over $A_{*}(n)$. Thus $H_{*}(X \wedge F)$ is free over the Steenrod algebra on a finite set of generators, whence $X \wedge F$ is a wedge of mod- $p$ Eilenberg-Mac Lane spectra $H \mathbb{F}_{p}$.

To show that 4 implies 1 , note that if $\pi_{*} Y$ is finite for a spectrum $Y$, then $Y$ can be built from finitely many copies of $H \mathbb{F}_{p}$, whence $H_{*} Y$ is finitely presented by (2.2). Thus if $\pi_{*}(X \wedge F)$ is finite, then $H_{*}(X \wedge F) \approx H_{*} X \otimes H_{*} F$ is finitely presented, and hence $H_{*} X$ is finitely presented by $(2.3)$. 
We call a spectrum $X$ an fp-spectrum if it is connective, $p$-complete, and satisfies any of the four equivalent statements of (3.2). Let $\mathcal{C}$ denote the class of all fp-spectra. This class includes the Eilenberg-Mac Lane spectra $H \mathbb{Z} / p^{n}$ and $H \mathbb{Z}_{p}$, the $p$-completed Johnson-Wilson spectrum $B P\langle n\rangle$, which has $\pi_{*} B P\langle n\rangle \approx$ $\mathbb{Z}_{p}\left[v_{1}, \ldots, v_{n}\right]$, and connective Morava $K$-theories. Non-trivial suspension spectra, and in particular finite complexes, are not fp-spectra.

Recall that a finite complex $F$ is of type $n$ if $K(0)_{*} F \approx \cdots \approx K(n-1)_{*} F \approx 0$ and $K(n)_{*} F \not 0$, where $K(m)$ denotes the $m$ th Morava $K$-theory. Define the fp-type of an fp-spectrum $X$ by

$$
\text { fptype }(X)=\min \left\{(\operatorname{typ}(F)-1) \text { such that } \pi_{*}(X \wedge F) \text { is finite }\right\} \text {. }
$$

By the thick subcategory theorem [6], if fptype $(X)=n$ then $\pi_{*}(X \wedge F)$ is finite for all $F$ of type $>n$. Thus, fptype $\left(H \mathbb{F}_{p}\right)=-1, \operatorname{fptype}\left(H \mathbb{Z}_{p}\right)=0$, and fptype $(B P\langle n\rangle)=n$. Furthermore, if $H_{*} X \approx A_{*} \otimes_{A_{*}(n)} M$, then fptype $(X) \leq n$.

Let $\mathcal{C}_{n}$ denote the class of fp-spectra of type $\leq n$. Then $\mathcal{C}_{n}$ is a subcategory of the category of spectra, and $\mathcal{C}_{n} \subset \mathcal{C}_{n+1}$.

Proposition 3.3. The classes $\mathcal{C}$ and $\mathcal{C}_{n}$ for $n \geq-1$ are thick subcategories of the homotopy category of spectra.

Proof. That $\mathcal{C}$ is a thick subcategory is an immediate consequence of criterion 4 of (3.2). Alternately, this follows from criterion 1 of (3.2) together with (2.2). The proof for $\mathcal{C}_{n}$ is similar.

For example, $\mathcal{C}_{-1}$ is the class of all $p$-complete spectra $X$ with $\pi_{*} X$ finite; as a thick subcategory it is generated by $H \mathbb{F}_{p}$. Likewise, $\mathcal{C}_{0}$ is the class of all fp-spectra which are finite Postnikov towers; as a thick subcategory it is generated by $H \mathbb{Z}_{p}$. The class $\mathcal{C}_{1}$ contains the $p$-completed connective $K$-theory spectra bo and $b u$, along with their connective covers. Thus $\mathcal{C}_{1}$ contains the image of $J$ spectrum, since $J \approx \mathrm{fib}($ bo $\rightarrow$ bspin $)$. The class $\mathcal{C}_{2}$ contains $e o_{2}$, the connective version of the "higher real $K$-theory" spectrum $E O_{2}$ of Hopkins and Miller.

3.4. $L_{n}$-localization and fp-spectra. Let $L_{n}$ denote Bousfield localization with respect to the wedge $K(0) \vee \cdots \vee K(n)$ of Morava $K$-theories. A spectrum $W$ is $L_{n}$-local if $L_{n} W \approx W$.

Proposition 3.5. Let $W$ be an $L_{n}$-local spectrum such that for each $k \in \mathbb{Z}$ the homotopy group $\pi_{k} W$ has the form

$$
\pi_{k} W \approx F_{k} \oplus \mathbb{Z}_{p}^{\oplus a_{k}} \oplus\left(\mathbb{Q} / \mathbb{Z}_{(p)}\right)^{\oplus b_{k}} \oplus \mathbb{Q}_{p}^{\oplus c_{k}},
$$

where $F_{k}$ is a finite p-group, $a_{k}=0=c_{k}$ for all sufficiently small $k \ll 0$, and $b_{k}=0=c_{k}$ for all sufficiently large $k \gg 0$.

Then there exists a map $f: X \rightarrow W$ such that $X$ is an fp-spectrum of fp-type $n$ and $L_{n} X \rightarrow L_{n} W \approx W$ is a weak equivalence.

Proof. Consider the connected cover $Y=W(-N, \ldots, \infty)$, where $N$ is chosen so that $a_{k}=c_{k}=0$ for $k<-N$. If $F$ is a finite complex with bottom cell in dimension 0 and top cell in dimension $d$, then the map $Y \wedge F \rightarrow W \wedge F$ is an isomorphism on $\pi_{k}$ for $k>d-N$, as can be seen by comparing the Atiyah-Hirzebruch spectral sequences computing $Y_{*} F$ and $W_{*} F$.

If $F$ is a type $(n+1)$ complex, then $W \wedge F \approx *$, and so $Y \wedge F$ has non-trivial homotopy in only a finite range of dimensions $(-N, \ldots, d-N)$, and each homotopy 
group is finite. Thus we have found a connective spectrum $Y$ which satisfies criterion 4 of (3.2), and furthermore $L_{n} Y \approx W$, since the fiber of $Y \rightarrow W$ is coconnective with torsion homotopy and thus is killed by $L_{n}$.

In order to get a $p$-complete spectrum, it suffices to replace any copies of $\mathbb{Q} / \mathbb{Z}_{(p)}$ or $\mathbb{Q}_{p}$ in the homotopy of $Y$ by a finite torsion group or a copy of $\mathbb{Z}_{p}$ respectively; by hypothesis there are only finitely many such copies to worry about. Note that $\left[\Sigma^{-i} H A, H \mathbb{Q} / \mathbb{Z}_{(p)}\right]$ is a finite torsion group if $A$ is a finitely generated $\mathbb{Z}_{p}$-module and $i>0$; thus by induction on the Postnikov tower of $Y$ we can see that we can always find a map

$$
Y \rightarrow \bigvee_{i=1}^{r} \Sigma^{n_{i}} H \mathbb{Q} / \mathbb{Z}_{(p)}
$$

which is surjective on homotopy, and so that the fiber $X$ of this map is $p$-complete. Then since $L_{n} H \mathbb{Q} / \mathbb{Z}_{(p)} \approx *$ we see that $X$ is the desired spectrum.

Remark 3.6. In view of Conjecture (7.3) and of (8.9), it seems likely that the converse of (3.5) should hold. That is, if $X$ is an fp-spectrum with fptype $(X)=n$, then we expect that $\pi_{k} L_{n} X$ has the form given in (3.5).

It is interesting to know when the $L_{n}$-localization of a finite complex $F$ is also the $L_{n}$-localization of an fp-spectrum $X$. One can say the following.

Proposition 3.7. If $F$ is the p-completion of a finite complex, then $L_{n} F$ is the $L_{n}$-localization of an fp-spectrum $X$ in the following cases,

1. $n=0$ or $n=1$ (at any prime),

2. $n=2$ if $p \geq 5$, or

3. for any $n$ and any prime $p$ if $F$ is a type $n$ complex.

Proof. It suffices to show in each case that the hypotheses of (3.5) are satisfied.

The cases $n=0$ and $n=1$ are well known. In fact, for $n=0$ take $X=H \mathbb{Z}_{p} \wedge F$, and for $n=1$ take $X=J \wedge F$, where $J$ is the connective image-of- $J$ spectrum.

If $n$ is arbitrary, but $F$ is a type $n$ complex, then the hypotheses of (3.5) hold. This is a consequence of the fact that the cohomology of the Morava stabilizer algebra is a finitely generated algebra (see [15, Thm. 6.2.10]), together with Hopkins and Ravenel's demonstration of a horizontal vanishing line at the $E_{\infty}$-term of the Adams-Novikov spectral sequence of $L_{n} F$ (see [16, Section 8.3]). These imply that $\pi_{k} F$ is finite for all $k$.

When $n=2$ and $p \geq 5$, one can take a spectrum of the form $Y \wedge F$, where $Y$ is an fp-spectrum such that $L_{2} Y \approx L_{2} S^{0}$. We show that the hypotheses of (3.5) hold for $L_{2} S^{0}$ at $p \geq 5$.

First, we note that the hypotheses of (3.5) hold for $L_{2} M(p)$, the localization of the $\bmod p$ Moore space. This is a consequence of calculations of Shimomura [18], as we explain below. There is a diagram

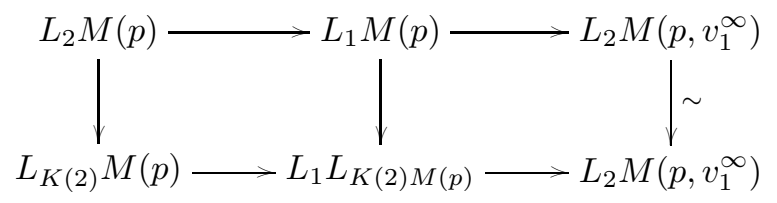

in which the left-hand square is a pull-back square; this is because all the objects in it are $L_{2}$-local, and the square is a pull-back after smashing with $K(0) \vee K(1) \vee K(2)$. 
Since $L_{1} M(p) \approx L_{1}\left(v_{1}^{-1} M(p)\right) \approx L_{2}\left(v_{1}^{-1} M(p)\right)$, the top row is a cofiber sequence, and thus so is the bottom row.

Shimomura computes the $E_{2}$-term of the Adams-Novikov spectral sequence for $L_{2} M\left(p, v_{1}^{\infty}\right)$. Using Shimomura's calculation one may (with careful analysis) read off that each group $\pi_{k} L_{2} M\left(p, v_{1}^{\infty}\right)$ must be finite; see the presentation of the results of this calculation given in [17]. Since $\pi_{k} L_{1} M(p)$ is known to be finite, this shows that $\pi_{k} L_{2} M(p)$ must be finite.

Hovey and Strickland [8, Thm. 15.1] actually carry out the careful analysis to show that each group $\pi_{k} L_{K(2)} M(p), k \in \mathbb{Z}$, is finite, so we will derive what we need from their results. To derive the finiteness of $\pi_{k} L_{2} M(p)$, it suffices to show that $L_{1} L_{K(2)} M(p)$ has finite homotopy groups. This spectrum is equivalent to $v_{1}^{-1} L_{K(2)} M(p)$, since the $v_{1}$-self map of $M(p)$ is trivial on $K(2)_{*} M(p)$. It happens that $\pi_{*} L_{K(2)} M(p)$ decomposes as a finite sum of copies $\mathbb{F}_{p}\left[v_{1}\right]$ plus a summand which is $v_{1}$-torsion. Thus $\pi_{*} v_{1}^{-1} L_{K(2)} M(p)$ is a finite sum of copies of the form $\mathbb{F}_{p}\left[v_{1}^{ \pm}\right]$, which is clearly a finite group in each dimension.

When $n=2$ and $p \geq 5$, the hypotheses of (3.5) hold for $L_{2} S^{0}$; this is a consequence of the above remarks together with the calculation of Shimomura and Yabe [19] of $\pi_{*} L_{2} S^{0}$ at $p \geq 5$. They show that the homotopy of $L_{2} S^{0}$ consists of a free summand in dimension 0 , summands of the form $\mathbb{Q} / \mathbb{Z}_{(p)}$ in stems $-3,-4$, and -5 , together with a summand $T$ consisting of non-infinitely divisible torsion. The above remarks on the finiteness of $\pi_{k} L_{2} M(p)$ imply that the summand $T$ of $\pi_{*} L_{2} S^{0}$ is finite in each stem.

We are led to make the following conjectures.

Conjecture 3.8. The hypotheses of (3.5) hold for any $L_{n}$-localization of a finite complex, for any $n \geq 0$.

Conjecture 3.9. For every finite complex $F$ there exists an fp-spectrum $X$ such that $L_{n} F \approx L_{n} X$.

Of course, Conjecture (3.8) implies Conjecture (3.9) as we have shown above. There is also reason to believe that Conjecture (3.9) would imply Conjecture (3.8); see section (7.2).

3.10. Adams towers for fp-spectra. For a spectrum $X$ we can construct an Adams tower. This is a tower of spectra $\ldots \rightarrow X_{s+1} \rightarrow X_{s} \rightarrow \ldots$ with $X_{0}=X$ and with fiber sequences $X_{s+1} \rightarrow X_{s} \stackrel{k_{s}}{\longrightarrow} \Sigma^{-s} H V_{s}$, where $k_{s}$ is injective on $\bmod p$ homology; hence there is a resolution

$$
0 \rightarrow H_{*} X \rightarrow H_{*} H V_{\bullet}
$$

and an Adams spectral sequence with $E_{2}^{s, t}=\operatorname{Ext}_{A_{*}}^{s, t}\left(\mathbb{F}_{p}, H_{*} X\right)$.

If $X$ is an fp-spectrum, we can choose an Adams tower in which each $V_{s}$ is a finite dimensional graded vector space. We call such an fp-Adams resolution. In fact, if $H_{*} X \approx A \otimes_{A_{*}(n)} M$ for some finite $A_{*}(n)$-module $M$, then the chain complex $H_{*} H V_{\bullet}$ is induced from a resolution of $M$ by $A_{*}(n)$-comodules.

Given an Adams tower $\left\{X_{s}\right\}$, we may produce another tower

$$
\ldots \rightarrow X_{0}^{s} \rightarrow X_{0}^{s-1} \rightarrow \ldots \rightarrow X_{0}^{1} \rightarrow X_{0}^{0} \rightarrow *
$$

by taking cofibers $X_{s+1} \rightarrow X \rightarrow X_{0}^{s}$. The fibers in this tower are $\Sigma^{-s} H V_{s} \rightarrow$ $X_{0}^{s} \rightarrow X_{0}^{s-1}$. Note that in the $E_{2}$-term of the spectral sequence for this tower, each 
horizontal line $E_{2}^{s, *}$ is finite dimensional, and thus the spectral sequence satisfies the complete convergence condition of [2, p. 263]. Thus the homotopy inverse limit $\operatorname{holim}_{s} X_{0}^{s}$ does not depend on the choice of fp-Adams resolution, and by a result of Bousfield [1, Prop. 5.8 and Thm. 6.6] is equivalent to $X$, since $X$ is $p$-complete and connective.

\section{Duality for finitely PRESEnted Comodules}

In this section we describe a duality functor $\tilde{I}$ on the category of finitely presented comodules. This functor was essentially introduced by Brown and Comenetz in [3]. Our interest in this functor stems from the fact that it generalizes the notion of "Spanier-Whitehead duality" of finite comodules over $A_{*}(n)$, in which a comodule is dual to its vector space dual. It will be needed in later sections to study duality on fp-spectra.

4.1. Construction of functors $\tilde{J}$ and $\tilde{I}$. To motivate the construction, note that if $X$ is a spectrum and $H$ denotes the mod $p$ Eilenberg-Mac Lane spectrum, then the graded vector space $[H, X]_{*}$ is in a natural way a module over the Steenrod algebra. This object is approximated by the edge map $[H, X]_{*} \rightarrow \operatorname{hom}_{A_{*}}\left(H_{*} H, H_{*} X\right)$ of the Adams spectral sequence. This algebraic approximation itself admits an action by the Steenrod action, and we will call this module $\tilde{J}\left(H_{*} X\right)$. The vector space dual to $\tilde{J}\left(H_{*} X\right)$ will be $\tilde{I}\left(H_{*} X\right)$, which admits a coaction by the dual Steenrod algebra.

Recall that if we regard the dual Steenrod algebra $A_{*}$ as a left-comodule over itself, then each element $a \in A^{*}$ of the Steenrod algebra induces a map $A_{*} \rightarrow A_{*}$ of left $A_{*}$-comodules via

$$
a \cdot z=\sum(-1)^{|a||z|} z^{\prime}\left\langle z^{\prime \prime}, a\right\rangle
$$

where $z \in A_{*}, \sum z^{\prime} \otimes z^{\prime \prime}$ is the diagonal of $z$ in $A_{*} \otimes A_{*}$, and $\langle z, a\rangle$ represents the usual pairing of $A_{*}$ and $A^{*}$. In fact, there is an isomorphism of algebras

$$
\operatorname{hom}_{A_{*}}\left(A_{*}, A_{*}\right) \approx A^{*} \text {. }
$$

We reserve the notation $a \cdot z$ for this action. Note that this action gives $A_{*}$ the structure of a left $A^{*}$-module; however, this is not identical to the usual left action of the Steenrod algebra on $H_{*} H$.

We define a functor $\tilde{J}$ from left $A_{*}$-comodules to left $A^{*}$-modules by

$$
\tilde{J}(M)=\operatorname{hom}_{A_{*}}\left(A_{*}, M\right) .
$$

This has a natural right $A^{*}$-action induced by pre-composition of comodule maps, using (4.2), which is made into a left $A^{*}$-action using $\chi$; if $z \in A_{*}, a \in A^{*}$, and $f \in \tilde{J}(M)$, the left action can be written

$$
(a \cdot f)(z)=(-1)^{|a||f|} f(\chi a \cdot z) .
$$

Note that if $M$ is finitely generated then $\tilde{J}(M)$ is bounded below (where we use cohomological grading for $\widetilde{J}(M)$ ).

We define a functor $\tilde{I}$ from finitely generated left comodules to left comodules by

$$
\tilde{I}(M)=(\tilde{J}(M))^{r} \approx \operatorname{hom}_{A_{*}}\left(A_{*}, M\right)^{r} .
$$

Since $\tilde{J}(M)$ was bounded below, $\tilde{I}(M)$ is bounded below, and thus receives a left comodule structure in the usual way. This structure is characterized as follows: if 
$u \in \tilde{I}(M)$ and $u \mapsto \sum u^{\prime} \otimes u^{\prime \prime} \in A_{*} \otimes \tilde{I}(M)$ is the comodule action on $u$, and if $a \in A^{*}$ and $f \in \tilde{J}(M)$, then

$$
\langle u, a \cdot f\rangle=\sum(-1)^{|a|\left|u^{\prime \prime}\right|}\left\langle u^{\prime}, a\right\rangle\left\langle u^{\prime \prime}, f\right\rangle .
$$

In comparison, Brown and Comenetz [3] define a functor $c_{p}$ on the category of $A^{*}$-modules; their functor is defined by

$$
c_{p}(M) \approx \operatorname{hom}_{A^{*}}\left(M, A^{*}\right),
$$

with an appropriate $A^{*}$-action. Thus, our $\tilde{I}$ is just a comodule version of their $c_{p}$.

4.3. Action of $\tilde{J}$ and $\tilde{I}$ on free comodules. Let $\mathcal{M}_{\text {free }} \subset \mathcal{M}_{\mathrm{fp}}$ denote the full subcategory of comodules of the form $A_{*} \otimes V$, where $V$ is a finite vector space. We want compute the action of $\tilde{J}$ and $\tilde{I}$ on this subcategory.

The following describes the category $\mathcal{M}_{\text {free }}$.

Proposition 4.4. For finite $V$ and $W$, $\operatorname{hom}_{A_{*}}\left(A_{*} \otimes V, A_{*} \otimes W\right) \approx A^{*} \otimes \operatorname{hom}(V, W)$, where $a \otimes \sigma \in A^{*} \otimes \operatorname{hom}(V, W)$ corresponds to the morphism of comodules

$$
z \otimes v \mapsto(-1)^{|\sigma||z|} a \cdot z \otimes \sigma(v), \quad z \in A_{*}, a \in A^{*}, v \in V, \sigma \in \operatorname{hom}(V, W),
$$

and composition of maps is given by $(a \otimes \sigma) \circ(b \otimes \tau)=(-1)^{|\sigma||b|} a b \otimes \sigma \tau$.

Proof. Straightforward.

Proposition 4.5. There is a natural isomorphism

$$
\tilde{J}\left(A_{*} \otimes V\right) \approx \operatorname{hom}_{A_{*}}\left(A_{*}, A_{*} \otimes V\right) \approx A^{*} \otimes V
$$

of left $A^{*}$-modules, where $a \otimes v \in A^{*} \otimes V$ corresponds to the map defined by

$$
z \mapsto(-1)^{|z||v|} \chi a \cdot z \otimes v, \quad z \in A_{*}, a \in A^{*}, v \in V .
$$

Given a map $a \otimes \sigma: A_{*} \otimes V \rightarrow A_{*} \otimes W$ of comodules, the induced map $\tilde{J}(a \otimes$ $\sigma): A^{*} \otimes V \rightarrow A^{*} \otimes W$ sends $b \otimes v \mapsto(-1)^{|b|(|a|+|\sigma|)} b \chi a \otimes \sigma(v)$.

Proof. Straightforward.

Corollary 4.6. There is a natural isomorphism

$$
\tilde{I}\left(A_{*} \otimes V\right) \approx\left(A^{*} \otimes V\right)^{\check{2}} \approx A_{*} \otimes \check{V}
$$

of left-comodules. The induced map $\tilde{I}(a \otimes \sigma): A_{*} \otimes \check{W} \rightarrow A_{*} \otimes \check{V}$ sends

$$
z \otimes \check{w} \mapsto(-1)^{|\sigma||z|} \chi a \cdot z \otimes \check{\sigma}(\check{w}), \quad z \in A_{*}, a \in A^{*}, \check{w} \in \check{W},
$$

where $\check{\sigma} \in \operatorname{hom}(\check{W}, \check{V})$ is the adjoint to $\sigma \in \operatorname{hom}(V, W)$. In other words, $\tilde{I}(a \otimes \sigma)=$ $\chi a \otimes \check{\sigma}$.

Remark 4.7. In terms of bases $v_{j}$ and $w_{i}$ for $V$ and $W$ we can view maps $A_{*} \otimes V \rightarrow$ $A_{*} \otimes W$ as matrices $\left(a_{i j}\right)$ with entries in the Steenrod algebra, acting by

$$
\sum_{j} z_{j} \otimes v_{j} \mapsto \sum_{i, j} a_{i j} \cdot z_{j} \otimes w_{i}
$$

Hence, the induced map $\tilde{I}\left(a_{i j}\right): A_{*} \otimes \check{W} \rightarrow A_{*} \otimes \check{V}$ corresponds to the matrix $\left(\chi a_{j i}\right)$ in terms of the dual bases $\check{v}_{j}$ and $\check{w}_{i}$ of $\check{V}$ and $\check{W}$. 


\subsection{Properties of the duality functor.}

Proposition 4.9. The functor $\tilde{I}$ restricts to a functor $\tilde{I}: \mathcal{M}_{\mathrm{fp}}^{\mathrm{op}} \rightarrow \mathcal{M}_{\mathrm{fp}}$, and is exact on $\mathcal{M}_{\mathrm{fp}}$. Furthermore, there is a natural isomorphism $M \rightarrow \tilde{I} \tilde{I} M$ for objects in $\mathcal{M}_{\mathrm{fp}}$.

Proof. That $\tilde{I}$ is exact on the subcategory of finitely presented comodules follows from (2.6). By (4.6) the functor $\tilde{I}$ takes finitely generated free comodules to the same, and thus by exactness $\tilde{I}\left(\mathcal{M}_{\mathrm{fp}}\right) \subset \mathcal{M}_{\mathrm{fp}}$.

One can construct a natural isomorphism $M \rightarrow \tilde{I} \tilde{I} M$ when $M \approx A_{*} \otimes V$, since by (4.6) $\tilde{I} \tilde{I}\left(A_{*} \otimes V\right)$ is tautologically isomorphic to $A_{*} \otimes V$. This isomorphism extends by exactness to all finitely presented comodules.

Proposition 4.10. Let $M$ be a finite $A_{*}(n)$-comodule. Then

$$
\tilde{I}\left(A_{*} \otimes_{A_{*}(n)} M\right) \approx A_{*} \otimes_{A_{*}(n)} \Sigma^{a(n)} \check{M}
$$

as left comodules, where $a(n)$ is the dimension of the "top cell" of $A_{*}(n)$.

Proof. There is an exact sequence

$$
0 \rightarrow M \rightarrow A_{*}(n) \otimes V \stackrel{\left(a_{i j}\right)}{\longrightarrow} A_{*}(n) \otimes W
$$

of $A_{*}(n)$-comodules, where $a_{i j} \in A^{*}(n)$. After applying vector space duals we can identify the resulting sequence with

$$
0 \leftarrow \Sigma^{-a(n)} \check{M} \leftarrow A_{*}(n) \otimes \check{V} \stackrel{\left(\chi a_{j i}\right)}{\longleftarrow} A_{*}(n) \otimes \check{W}
$$

by "Poincaré duality" of $A_{*}(n)$ [12, Ch. 12.2]. The result follows from (4.7) after extending up to $A_{*}$.

\section{Brown-Comenetz DUALity}

Recall that the functor

$$
X \mapsto \operatorname{hom}\left(\pi_{0} X, \mathbb{Q} / \mathbb{Z}\right)
$$

is a generalized cohomology theory satisfying the wedge axiom, and hence is represented by a spectrum $I$. We write $I Y=\mathcal{F}(Y, I)$ for the function spectrum, whence $I Y$ is the spectrum representing the functor

$$
X \mapsto I Y^{0}(X)=\operatorname{hom}\left(Y_{0} X, \mathbb{Q} / \mathbb{Z}\right) .
$$

The spectrum $I Y$ is called the Brown-Comenetz dual of $Y$.

We write $D X \approx \mathcal{F}\left(X, S^{0}\right)$ for the Spanier-Whitehead dual of $X$. Note that if $X$ is any spectrum and $F$ is a finite complex, then the natural map $I X \wedge D F \rightarrow I(X \wedge F)$ is an equivalence.

There is a natural double-dual map $X \rightarrow I I X$. If $Y$ is a spectrum such that each homotopy group $\pi_{k} Y$ is finite, then $Y \approx I I Y$ via this map. Thus, given such a $Y$ and given any spectrum $X$, there is a natural isomorphism

$$
[X, Y] \approx[I Y, I X] .
$$


5.1. Eilenberg-Mac Lane spectra. Let $H \approx H \mathbb{F}_{p}$ denote the $\bmod p$ EilenbergMac Lane spectrum. Then $I H \approx H$. In fact, by the universal coefficient theorem,

$$
I H^{*} X \approx \operatorname{hom}\left(H_{*} X, \mathbb{Q} / \mathbb{Z}\right) \approx \operatorname{hom}\left(H_{*} X, \mathbb{F}_{p}\right) \approx H^{*} X .
$$

Lemma 5.2. Under the above identification the map

$$
I:[H, H]_{*} \rightarrow[I H, I H]_{*} \approx[H, H]_{*}
$$

sends $a \in A^{*}$ to $\chi a$.

Proof. This is [3, Thm. 1.9(d)].

More generally, let $V$ denote a finite dimensional graded $\mathbb{F}_{p}$-vector space, and let $\check{V}=\operatorname{hom}\left(V, \mathbb{F}_{p}\right)$ denote its vector space dual. Let $H V$ denote the generalized Eilenberg-Mac Lane spectrum with $\pi_{*} H V=V$, whence $H V_{*} X \approx H_{*} X \otimes V$.

Proposition 5.3. There is an equivalence $I H V \approx H \check{V}$, and we have isomorphisms

$$
H^{*}(I H V) \approx \tilde{J}\left(H_{*} H V\right)
$$

of $A^{*}$-modules and

$$
H_{*}(I H V) \approx \tilde{I}\left(H_{*} H V\right)
$$

of $A_{*}$-comodules which are natural in $H V$.

Proof. This is immediate from (4.6) and (5.2); alternatively, it follows from [3, Thm. 1.3].

5.4. Algebraic approximation. We note that the functor $\tilde{I}$ of Section 4 serves as an algebraic "approximation" to $H_{*} I X$, at least when $H_{*} X$ has finitely presented homology.

Proposition 5.5. For each spectrum $X$ with finitely presented homology, there is a map

$$
\iota_{X}: \tilde{I}\left(H_{*} X\right) \rightarrow H_{*} I X
$$

which is natural in $X$. Furthermore, this map is an isomorphism when $\pi_{*} X$ is finite.

Compare with [3, Thm. 1.13].

Proof. Given $X$ we can choose an Adams resolution

$$
X \rightarrow H V_{0} \rightarrow H V_{1} \rightarrow \ldots,
$$

in which $V_{0}$ and $V_{1}$ are finite dimensional vector spaces, and the sequence

$$
0 \rightarrow H_{*} X \rightarrow H_{*} H V_{0} \rightarrow H_{*} H V_{1}
$$

is exact. Applying $I$ to the first diagram gives maps

$$
I X \leftarrow H \check{V}_{0} \leftarrow H \check{V}_{1}
$$

and a sequence

$$
H_{*} I X \leftarrow \tilde{I}\left(H_{*} H V_{0}\right) \leftarrow \tilde{I}\left(H_{*} H V_{1}\right),
$$

not necessarily exact. We let $\iota_{X}$ be the induced map

$$
\iota: \tilde{I}\left(H_{*} X\right)=\operatorname{Cok}\left(\tilde{I}\left(H_{*} H V_{1}\right) \rightarrow \tilde{I}\left(H_{*} H V_{0}\right)\right) \rightarrow H_{*} I X .
$$

To see that $\iota_{X}$ is independent of the choice of resolution and is natural, use a map between Adams resolutions. 
The map $\iota$ is by construction an isomorphism for $X=H \mathbb{F}_{p}$. By the exactness property of (4.9), we see that $\iota_{X}$ is an isomorphism for any $X$ in the thick subcategory generated by $H \mathbb{F}_{p}$, which are precisely the $X$ with finite homotopy.

\section{Geometric realization of Tate cohomology}

In this section we note that one can construct for each fp-spectrum a $\mathbb{Z}$-indexed Adams tower; this is a tower which extends both above and below $X$, whose layers are finite mod $p$ generalized Eilenberg-Mac Lane spectra, and which leads to a spectral sequence whose $E_{2}$-term is the Tate cohomology of $H_{*} X$. We give several constructions, starting with the most general.

\subsection{Construction of the tower.}

Lemma 6.2. Let $X$ be an fp-spectrum. Then

$$
[H, X]_{*} \approx \operatorname{hom}_{A_{*}}\left(A_{*}, H_{*} X\right) \approx \tilde{J}\left(H_{*} X\right) .
$$

Proof. Choose an fp-Adams tower $\left\{X_{s}\right\}$ for $X$. Then there is a spectral sequence $E_{1}^{s, t}=\left[H, H V_{s}\right]_{t} \Longrightarrow[H, X]_{t-s}$. We claim that

1. $E_{2}^{s, t} \approx 0$ for $s>0$, so that $E_{2}^{0, t} \approx E_{\infty}^{*, t}$, and

2. $E_{2}^{0, t} \approx \operatorname{hom}_{A_{*}}\left(A_{*}, H_{*} X\right)$.

Since $X$ is connective and $p$-complete, the first claim implies that the spectral sequence converges, and thus $E_{2}^{0, t} \approx[H, X]_{t}$.

To prove the claim, recall that the resolution $0 \rightarrow H_{*} X \rightarrow H_{*} H V_{s}$ is extended up from a resolution $0 \rightarrow M \rightarrow C(s)$ of $A_{*}(n)$-modules. Now

$$
\left[H, H V_{s}\right]_{*} \approx \operatorname{hom}_{A_{*}}\left(A_{*}, A_{*} \otimes_{A_{*}(n)} C(s)\right) \approx \operatorname{hom}_{A_{*}(n)}\left(A_{*}, C(s)\right) .
$$

Since as an $A_{*}(n)$-comodule $A_{*} \approx A_{*}(n) \otimes A_{*} / / A_{*}(n)$ we see that $A_{*}$ is projective as an $A_{*}(n)$-comodule, and thus the sequence

$$
0 \rightarrow \operatorname{hom}_{A_{*}}\left(A_{*}, H_{*} X\right) \rightarrow \operatorname{hom}_{A_{*}}\left(A_{*}, H_{*} H V_{\bullet}\right)
$$

is exact as desired.

Thus, given an fp-spectrum $X$, we may construct a tower "realizing" the Tate cohomology by constructing fp-spectra $X_{-s}$ for $s \geq 0$ inductively as follows. First, let $X_{0}=X$. Since $H_{*} X_{-s} \approx A_{*} \otimes_{A_{*}(n)} M(-s)$ for some finite module $M(-s)$, we can choose a surjection $C(-s-1) \rightarrow M(-s)$ from a free $A_{*}(n)$-comodule. This may be extended to a surjection $A_{*} \otimes_{A_{*}(n)} C(-s-1) \rightarrow A_{*} \otimes_{A_{*}(n)} M(-s)$, which in turn by the above lemma is realized by a map of spectra $\Sigma^{s} H V_{-s-1} \rightarrow X_{-s}$. If we take the cofiber

$$
\Sigma^{s} H V_{-s-1} \rightarrow X_{-s} \rightarrow X_{-s-1}
$$

we get another fp-spectrum $X_{-s-1}$; iteration produces an infinite sequence $\ldots \rightarrow$ $X_{-s} \rightarrow X_{-s-1} \rightarrow \ldots$ If we put this sequence together with an fp-Adams tower for $X$, we get a tower $\left\{X_{s}\right\}_{s \in \mathbb{Z}}$, which we call a $\mathbb{Z}$-indexed Adams tower for $X$.

Note that given a map $f: X \rightarrow Y$ of fp-spectra and given $\mathbb{Z}$-indexed Adams towers $\left\{X_{s}\right\}$ and $\left\{Y_{s}\right\}$, we can extend $f$ to a map of towers, by the "dual" of the usual argument, using (6.2). In particular, given any two $\mathbb{Z}$-indexed Adams towers for $X$ we can produce a map between them.

For such a tower, let $\widehat{X}=$ hocolim $_{s \rightarrow \infty} X_{-s}$. There is a full-plane Adams-type spectral sequence $E_{2}^{s, t} \approx H_{\text {Tate }}^{s, t}\left(H_{*} X\right) \Longrightarrow \pi_{t-s} \widehat{X}$ approximating the homotopy of $\widehat{X}$, where $H_{\text {Tate }}^{s, t}$ is the Tate cohomology of (2.5). 
Given such a tower $\left\{X_{s}\right\}$, write $X_{p}^{q}=\operatorname{cof}\left(X_{q+1} \rightarrow X_{p}\right)$ for $-\infty<p \leq q<\infty$. Then let $X_{\infty}^{-1}=\operatorname{cof}(X \rightarrow \widehat{X}) \approx \operatorname{hocolim}_{s \rightarrow \infty} X_{-s}^{-1}$. A straightforward convergence argument shows that the homotopy spectral sequence of this colimit converges, and hence $X_{\infty}^{-1}$ does not depend on the choice of the $\mathbb{Z}$-indexed tower.

The above remarks are summarized in the following proposition.

\section{Proposition 6.3.}

1. For each fp-spectrum $X$ there exists a $\mathbb{Z}$-indexed Adams tower $\left\{X_{s}\right\}$.

2. Given $\mathbb{Z}$-indexed Adams towers $\left\{X_{s}\right\}$ and $\left\{Y_{s}\right\}$ for $X$ and $Y$, and a map $f: X \rightarrow Y$, there exists a map $\left\{f_{s}: X_{s} \rightarrow Y_{s}\right\}$ of towers which extends $f$.

3. The colimit $\widehat{X}=$ hocolim $_{s \rightarrow \infty} X_{-s}$ of a $\mathbb{Z}$-indexed Adams tower depends only on $X$, and not on the choice of tower.

6.4. Alternate construction of the tower. Let $X$ be an fp-spectrum, and let $F$ be a finite complex such that $H_{*}(X) \otimes H_{*}(F)$ is a free $A_{*}$-comodule. Suppose also a map $S^{0} \rightarrow F$ which is injective on homology. Such an $F$ always exists; if $H_{*} X$ is an extended $A_{*}(n)$-comodule, let $F$ be a Mitchell complex of type $n$ as in (3.1), and $S^{0} \rightarrow F$ the inclusion of a bottom cell.

Form a fiber sequence $\bar{F} \rightarrow S^{0} \rightarrow F$, so that by Spanier-Whitehead duality we get a dual fiber sequence $D F \rightarrow S^{0} \rightarrow D \bar{F}$. We obtain a $\mathbb{Z}$-indexed Adams tower with

$$
X_{s}= \begin{cases}X \wedge \bar{F}^{(s)} & \text { if } s \geq 0 \\ X \wedge(D \bar{F})^{(-s)} & \text { if } s<0,\end{cases}
$$

where the maps in the tower are induced by fiber sequences $X \wedge \bar{F}^{(s+1)} \rightarrow X \wedge \bar{F}^{(s)} \rightarrow$ $X \wedge \bar{F}^{(s)} \wedge F$ and $X \wedge D \bar{F}^{(s)} \wedge D F \rightarrow X \wedge D \bar{F}^{(s)} \rightarrow X \wedge D \bar{F}^{(s+1)}$.

In those cases when there actually exists a complex $F$ with $H_{*} F \approx A_{*}(n)$, and $H_{*} X$ is an extended $A_{*}(n)$-module, then this complex realizes the unbounded chain complex obtained by gluing together the bar complex for $M$ and the co-bar complex for $M$ as an $A_{*}(n)$-comodule.

6.5. An explicit construction for bo. Recall that $H^{*} b o \approx A^{*} / A^{*}\left(S q^{1}, S q^{2}\right)$. Consider the minimal Adams tower $\left\{b o^{s}\right\}$ for bo at $p=2$; write $H^{*} b o^{s} \approx A^{*} \otimes_{A^{*}(1)}$ $M(s)$.

Let $R(n)$ denote the cofiber of $P^{n} \rightarrow S^{0}$ for $n \geq 0$; thus $R(0) \approx S^{0}$. Recall from [9] that

$$
M(4 s) \approx H^{*} R(8 s) .
$$

We define a map $R(8 s+8) \rightarrow R(8 s)$ as follows. Let $p: R(8 s+8) \rightarrow \Sigma P_{8 s+1}^{8 s+8}$ denote the pinch map obtained by taking the quotient of $R(8 s) \rightarrow R(8 s+8)$. Then $p \circ(16) \sim 0$, where (16) is the degree 16 map on $R(8 s+8) \rightarrow R(8 s+8)$, and so (16) lifts to a map $f_{s}: R(8 s+8) \rightarrow R(8 s)$. The map $f_{s}$ has degree 16 on the bottom cell.

Likewise, we may consider the Spanier-Whitehead dual map

$$
f_{-s}=D f_{s-1}: D R(8 s-8) \rightarrow D R(8 s) .
$$

This map has degree 16 on the top cell. Note that $f_{-1}: S^{0} \rightarrow D R(8 s)$, so we can put all the $f_{s}$ for $s \in \mathbb{Z}$ together into a $\mathbb{Z}$-indexed tower

$$
\ldots \stackrel{f_{2}}{\longrightarrow} R(16) \stackrel{f_{1}}{\longrightarrow} R(8) \stackrel{f_{0}}{\longrightarrow} R(0) \approx S^{0} \stackrel{f_{-1}}{\longrightarrow} D R(8) \stackrel{f_{-2}}{\longrightarrow} D R(16) \stackrel{f_{-3}}{\longrightarrow} \ldots
$$


Proposition 6.6. There is a $\mathbb{Z}$-indexed Adams tower $\left\{b o^{s}\right\}$ for bo with

$$
b o^{4 s} \approx \begin{cases}b o \wedge R(8 s) & \text { if } s \geq 0, \\ b o \wedge D R(-8 s) & \text { if } s<0\end{cases}
$$

and the map $b o^{4 s+4} \rightarrow b o^{4 s}$ is bo $\wedge f_{s}$.

Proof. Set $b o^{4 s}$ as above, and construct a the start of a minimal Adams tower $b o^{4 s+3} \rightarrow b o^{4 s+2} \rightarrow b o^{4 s+1} \rightarrow b o^{4 s}$ over $b o^{s}$. It is not hard to see that $R(8 s+8) \rightarrow$ $b o \wedge R(8 s) \approx b o^{4 s}$ lifts to $b o^{4 s+3}$, so that we can extend to a map bo $\wedge R(8 s+8) \rightarrow$ $b o^{4 s+3}$; likewise, $D R(8 s-8) \rightarrow b o \wedge D R(8 s) \approx b o^{-4 s}$ lifts to $b o^{-4 s+3}$, so we can extend to a map $b o \wedge D R(8 s-8) \rightarrow b o^{-4 s+3}$. The resulting tower is an Adams tower.

Here is a chart which presents the Adams $E_{2}$-term for bo $\wedge D R(16)$.

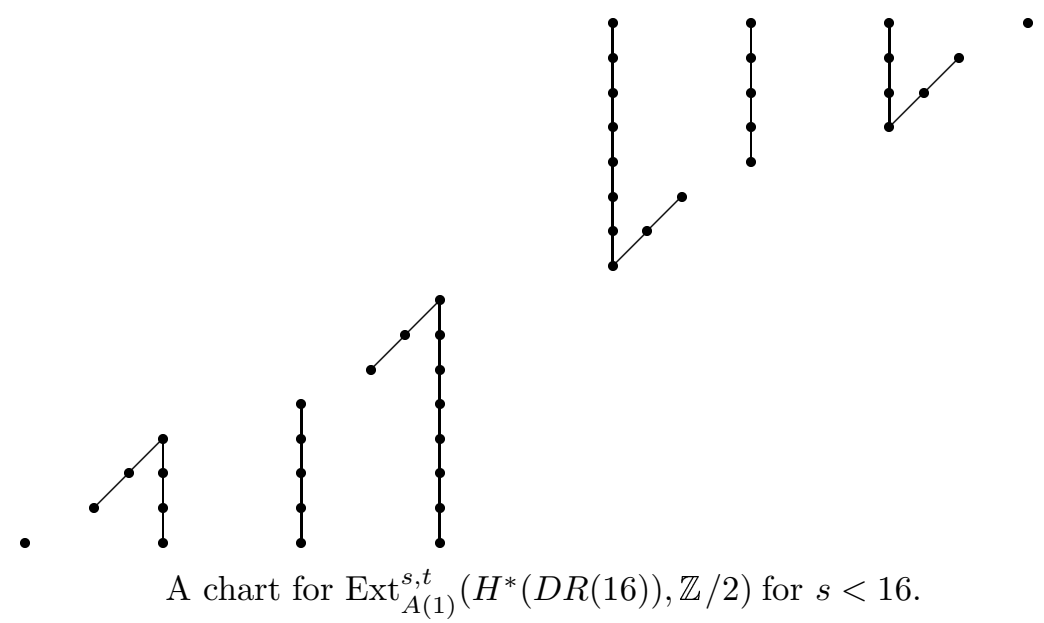

\section{Finite LOCALization}

There exists a functor $L_{n}^{f}$ and natural map $X \rightarrow L_{n}^{f} X$, called finite localization. It is Bousfield localization with respect to the wedge $\operatorname{Tel}(0) \vee \cdots \vee \operatorname{Tel}(n)$, where $\operatorname{Tel}(k)$ denotes the $v_{k}$-telescope on some chosen type $k$ finite complex. The functor $L_{n}^{f}$ is characterized by the following properties [11].

1. The fiber $C_{n}^{f} X=\mathrm{fib}\left(X \rightarrow L_{n}^{f} X\right)$ is a homotopy colimit of some diagram of type- $(n+1)$ finite complexes.

2. There are no essential maps from a type $(n+1)$ finite complex to $L_{n}^{f} X$ (i.e., $L_{n}^{f} X$ is $L_{n}^{f}$-local).

3. $L_{n}^{f} X$ is smashing; i.e., $L_{n}^{f} X \approx X \wedge L_{n}^{f} S^{0}$.

Note also that 1 and 2 imply that $L_{n}^{f} X \approx X$ if $X$ is $L_{n}^{f}$-local. Also, if $X$ is a type $n$ finite complex, then $L_{n}^{f} X \approx v^{-1} X$, where $v: \Sigma^{d} X \rightarrow X$ is a $v_{n}$-self map of $X$.

Theorem 7.1. Let $X$ be an fp-spectrum with fptype $(X) \leq n$, and let $\left\{X_{s}\right\}$ be a $\mathbb{Z}$-indexed Adams tower for $X$, with colimit $\widehat{X}$. Then $L_{n}^{f} X \approx \widehat{X}$.

Proof. Consider the map $i: X \rightarrow \widehat{X}$. It suffices to show that

1. $L_{n}^{f}(i)$ is an equivalence, and

2. $\widehat{X}$ is $L_{n}^{f}$-local. 
Claim 1 follows immediately from the fact that $L_{n}^{f} H \mathbb{F}_{p} \approx *$ and that $L_{n}^{f}$ is smashing, since $\widehat{X}$ is obtained from $X$ by attaching mod $p$ Eilenberg-Mac Lane spectra.

To prove claim 2, we must show that $\widehat{X}$ admits no essential maps from a type$(n+1)$ finite complex $F$, or equivalently, that $\widehat{X} \wedge F \approx *$ for any such complex. This follows from the following:

3. For any finite complex $F,\left\{X_{s} \wedge F\right\}$ is a $\mathbb{Z}$-indexed Adams tower for $X \wedge F$, and $\widehat{X} \wedge F \approx \operatorname{hocolim} X_{-s} \wedge F$.

4. If $Y$ is an fp-spectrum with $\pi_{*} Y$ finite, then $\widehat{Y} \approx *$ for any $\mathbb{Z}$-indexed Adams tower for $Y$.

The proof of claim 3 is straightforward.

To prove claim 4, apply Brown-Comenetz duality to the sequence

$$
Y=Y_{0} \rightarrow Y_{-1} \rightarrow Y_{-2} \rightarrow \ldots \rightarrow \widehat{Y} .
$$

This produces a tower

$$
I \widehat{Y} \rightarrow \ldots \rightarrow I Y_{-2} \rightarrow I Y_{-1} \rightarrow I Y_{0}=I Y
$$

which is easily seen to be an Adams tower for $I Y$ by (5.5). Since $I Y$ is connective and $p$-complete and $\pi_{*} I Y$ is finite, its Adams spectral sequence converges, and $I \widehat{Y} \approx *$, whence $\widehat{Y} \approx *$.

7.2. Relation between $L_{n}^{f}$ and $L_{n}$. Let $X \rightarrow L_{n} X$ denote Bousfield localization with respect to $K(0) \vee \cdots \vee K(n)$. There is a natural map $t_{n}: L_{n}^{f} X \rightarrow L_{n} X$; this map is an equivalence for $X \approx S^{0}$, and hence for all $X$, if and only if the Telescope Conjecture holds for all $m \leq n$. This conjecture is true for $n=0$ and $n=1$, and is believed to be false for $n \geq 2$.

It is reasonable to ask whether $t_{n}$ is an equivalence when $X$ is an fp-spectrum. We note that $t_{n}$ is an equivalence in the following cases, which include all cases we know of.

1. Since $B P\langle n\rangle$ is a $B P$-module spectrum, one can compute $L_{n} B P\langle n\rangle$ using the chromatic tower method of [14, Sec. 6]. (We would like to thank Hal Sadofsky for pointing this out to us.) This calculation shows in particular that the fiber of $B P\langle n\rangle \rightarrow L_{n} B P\langle n\rangle$ is coconnective with torsion homotopy; thus the fiber is killed by $L_{n}^{f}$, and hence $t_{n}$ is an equivalence on $L_{n} B P\langle n\rangle$, and is in fact an equivalence on the thick subcategory of $\mathcal{C}$ generated by $B P\langle n\rangle$.

2. For any fp-spectrum $X$ obtained by the procedure of the proof of (3.5) the map $t_{n}: L_{n}^{f} X \rightarrow L_{n} X$ is an equivalence, since by construction the fiber of $X \rightarrow L_{n} X$ is coconnective with torsion homotopy.

We make the following conjecture.

Conjecture 7.3. The map $t_{n}: L_{n}^{f} X \rightarrow L_{n} X$ is an equivalence for all fp-spectra $X$.

This conjecture is of interest, because it would give information on how badly the Telescope Conjecture fails, assuming it does fail. Namely, suppose $F$ is a type $n$ finite complex and $v^{-1} F$ is its $v_{n}$-telescope; then if the Telescope Conjecture fails for $n$ and Conjecture (7.3) holds, it follows that $\pi_{k}\left(v^{-1} F\right)$ is an infinite group for some $k \in \mathbb{Z}$, whereas $\pi_{k} L_{n} F$ is finite for all $k$. To see this, we argue as follows; if each $\pi_{k}\left(v^{-1} F\right)$ were a finite group, then the proof of (3.5) would apply to show that there exists an fp-spectrum $X$ with $L_{n}^{f} X \approx v^{-1} F$. (We could take $X$ to 
be a connective cover of $v^{-1} F$, for example.) However, Conjecture (7.3) would then imply that $v^{-1} F \approx L_{n} X \approx L_{n} F$, contradicting the failure of the Telescope Conjecture.

Conjecture (7.3) also would imply, using (8.9) of the next section, that the two conjectures (3.8) and (3.9) discussed in section 3 are in fact equivalent statements.

\section{Duality}

\subsection{Duality for fp-spectra of type less than $n$. Let}

$$
W_{n} X=I C_{n}^{f} X
$$

where $C_{n}^{f}=\mathrm{fib}\left(X \rightarrow L_{n}^{f} X\right)$. Thus $W_{n}$ is a contravariant functor from spectra to spectra. Since $L_{n}^{f}$ is smashing,

$$
W_{n} X \approx \mathcal{F}\left(X \wedge C_{n}^{f} S^{0}, I S^{0}\right) \approx \mathcal{F}\left(X, W_{n} S^{0}\right) .
$$

That is, $W_{n} S^{0}$ is a "dualizing complex" for $W_{n}$. Furthermore, there is a natural map $X \rightarrow W_{n} W_{n} X$; this map is adjoint to the evaluation map $X \wedge \mathcal{F}\left(X, W_{n} S^{0}\right) \rightarrow$ $W_{n} S^{0}$.

Note that $W_{n}$ vanishes on $L_{n}^{f}$-local spectra. Also, if $\pi_{*} X$ is finite, then $L_{n}^{f} X \approx *$ and we have $W_{n} X \approx I X$.

Recall that $\mathcal{C}_{n}$ denotes the homotopy category of fp-spectra with fp-type $\leq n$.

Theorem 8.2. Let $X$ be an object in $\mathcal{C}_{n}$.

1. There is a natural isomorphism $H_{*} W_{n} X \approx \tilde{I}\left(H_{*} X\right)$.

2. $W_{n} X$ is in $\mathcal{C}_{n}$.

3. The natural map $X \rightarrow W_{n} W_{n} X$ is an equivalence.

From this we obtain the following.

Corollary 8.3. The functor $W_{n}$ restricts to a functor $W_{n}: \mathcal{C}_{n}^{\text {op }} \rightarrow \mathcal{C}_{n}$, which is an equivalence of categories.

Proof. The corollary follows from the fact that the functor $W_{n}$ is "self-adjoint"; that is,

$$
W_{n}: \mathcal{S}^{\mathrm{op}} \rightleftarrows \mathcal{S}:\left(W_{n}\right)^{\mathrm{op}}
$$

is a pair of adjoint functors, where $\mathcal{S}$ represents the homotopy category of spectra. Part 2 of (8.2) says that $W_{n}$ carries $\mathcal{C}_{n}^{\text {op }}$ into $\mathcal{C}_{n}$, and part 3 says that the restriction of $W_{n}$ to $\mathcal{C}_{n}$ gives an adjoint equivalence $W_{n}: \mathcal{C}_{n}^{\text {op }} \rightleftarrows \mathcal{C}_{n}:\left(W_{n}\right)^{\text {op }}$ of categories.

Proof of Theorem 8.2. If $X$ is an fp-spectrum, choose a $\mathbb{Z}$-indexed Adams tower $\left\{X_{s}\right\}$, and as in (6.1) write $X_{q}^{p}=\operatorname{cof}\left(X_{p+1} \rightarrow X_{q}\right)$. Then

$$
C_{n}^{f} X \approx \Sigma^{-1} \underset{s \rightarrow \infty}{\operatorname{hocolim}} X_{-s}^{-1}
$$

by (7.1), and thus

$$
W_{n} X \approx \Sigma\left(\operatorname{holim}_{s \rightarrow \infty} I X_{-s}^{-1}\right) .
$$

Write $H_{*} X \approx A_{*} \otimes_{A_{*}(n)} M$. We can construct the bottom part of the $\mathbb{Z}$-indexed tower to realize any projective resolution of $M$ by finite $A_{*}(n)$-modules, e.g., by the resolution dual to the minimal resolution $0 \rightarrow \check{M} \rightarrow C_{\bullet}$ of $\check{M}$ by finite $A_{*}(n)$ modules. Then (8.4) immediately implies that $W_{n} X$ is connective and $p$-complete, since the tower $\left\{I\left(X_{-s}^{-1}\right)\right\}_{s \geq 1}$ must necessarily be the tower associated with an 
Adams tower for $W_{n} X$ which realizes $C_{\bullet}$, and so the connectivity of $I\left(X_{-s}^{-1}\right)$ is bounded below by a fixed $N$ for all $s \geq 1$.

Note that for any $Y$ we have that $H \mathbb{F}_{p} \wedge L_{n}^{f} Y \approx *$, whence the map $H_{*} C_{n}^{f} Y \rightarrow$ $H_{*} Y$ is an isomorphism, so that there exists by (5.5) a natural comparison map

$$
\iota^{n}(Y): \tilde{I}\left(H_{*} Y\right) \stackrel{\sim}{\longrightarrow} \tilde{I}\left(H_{*} C_{n}^{f} Y\right) \rightarrow H_{*} W_{n} Y .
$$

We want to show that $\iota^{n}(X)$ is an isomorphism.

Since $X$ has a $\mathbb{Z}$-indexed tower, there is a sequence

$$
\ldots \rightarrow H V_{-2} \rightarrow H V_{-1} \rightarrow X
$$

which induces an exact sequence

$$
H_{*} H V_{-2} \rightarrow H_{*} H V_{-1} \rightarrow H_{*} X \rightarrow 0
$$

on homology. Applying $W_{n}$ to this sequence gives a sequence

$$
W_{n} X \rightarrow W_{n} H V_{-1} \rightarrow W_{n} H V_{-2} \rightarrow \ldots
$$

which corresponds to an Adams resolution for $W_{n} X$ and hence induces an exact sequence

$$
0 \rightarrow H_{*} W_{n} X \rightarrow H_{*} W_{n} H V_{-1} \rightarrow H_{*} W_{n} H V_{-2} .
$$

It is clear that the comparison maps $\iota^{n}\left(H V_{-s}\right)$ are isomorphisms, and so $\iota^{n}(X)$ is an isomorphism, since $\tilde{I}$ is exact. Thus we have proved parts 1 and 2 of the proposition.

Part 3 follows from parts 1 and 2, together with (8.7), to be proved below.

Remark 8.5. If $X$ is a spectrum which might not be $p$-complete, and $X_{p}$ denotes its $p$-completion, then one can show via an arithmetic square argument that $C_{n}^{f} X \approx$ $C_{n}^{f} X_{p}$, and hence $W_{n} X \approx W_{n} X_{p}$. In particular, if $X_{p}$ is an fp-spectrum, we may conclude that $W_{n} W_{n} X \approx X_{p}$.

We still owe the reader one more fact.

Proposition 8.6. Suppose $X$ and $W_{n} X$ have finitely presented homology. Then there is a commutative square

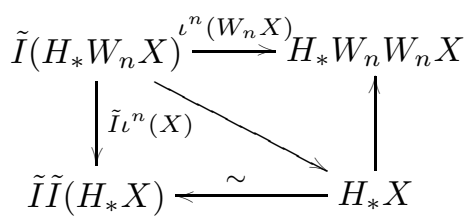

Corollary 8.7. If $X$ and $W_{n} X$ have finitely presented homology and the maps

$$
\iota^{n}(X): \tilde{I}\left(H_{*} X\right) \rightarrow H_{*} W_{n} X, \quad \text { and } \quad \iota^{n}\left(W_{n} X\right): \tilde{I}\left(H_{*} W_{n} X\right) \rightarrow H_{*} W_{n} W_{n} X
$$

are isomorphisms, then the the map $X \rightarrow W_{n} W_{n} X$ induces an isomorphism in homology.

Proof of Proposition 8.6. Choose resolutions $X \rightarrow H C_{0} \rightarrow H C_{1}$ and $W_{n} X \rightarrow$ $H D_{0} \rightarrow H D_{1}$. This leads to a sequence

$$
H \check{D}_{1} \rightarrow H \check{D}_{0} \rightarrow W_{n} W_{n} X \rightarrow H C_{0} \rightarrow H C_{1} .
$$

The diagonal map in the diagram is the induced map

$$
\operatorname{Cok}\left(H_{*} H \check{D}_{1} \rightarrow H_{*} H \check{D}_{0}\right) \rightarrow \operatorname{Ker}\left(H_{*} H C_{0} \rightarrow H_{*} H C_{1}\right) .
$$

It is straightforward to check commutativity of the diagram. 


\subsection{A finiteness result.}

Proposition 8.9. Let $X$ be an fp-spectrum with fptype $(X)=n$, and let $Y=L_{n}^{f} X$ be its finite localization. Then for each $k \in \mathbb{Z}$ the homotopy group $\pi_{k} Y$ has the form

$$
\pi_{k} Y \approx F_{k} \oplus \mathbb{Z}_{p}^{\oplus a_{k}} \oplus\left(\mathbb{Q} / \mathbb{Z}_{(p)}\right)^{\oplus b_{k}} \oplus \mathbb{Q}_{p}^{\oplus c_{k}},
$$

where $F_{k}$ is a finite p-group, $a_{k}=0=c_{k}$ for all sufficiently small $k \ll 0$, and $b_{k}=0=c_{k}$ for all sufficiently large $k \gg 0$.

Proof. There is a fiber sequence $C_{n}^{f} X \rightarrow X \rightarrow L_{n}^{f} X$, and the Brown-Comenetz dual $W_{n} X$ of $C_{n}^{f} X$ is an fp-spectrum by (8.2). Thus $X$ is connective with $\pi_{k} X \approx$ $F_{k} \oplus \mathbb{Z}_{p}^{m_{k}}$, and $C_{n}^{f} X$ is coconnective with $\pi_{k} C_{n}^{f} X \approx F_{k}^{\prime} \oplus\left(\mathbb{Q} / \mathbb{Z}_{(p)}\right)^{n_{k}}$. The image and coimage of the connecting map $\pi_{k} C_{n}^{f} X \rightarrow \pi_{k} X$ can only be a finite torsion group; thus, to prove the result for $\pi_{k} L_{n}^{f} X$ we need to show that, in a group extension of the form

$$
0 \rightarrow F \oplus \mathbb{Z}_{p}^{m} \rightarrow M \rightarrow F^{\prime} \oplus\left(\mathbb{Q} / \mathbb{Z}_{(p)}\right)^{n} \rightarrow 0,
$$

where $F$ and $F^{\prime}$ are finite $p$-groups, that $M$ is as described in the statement of the proposition.

It is easy to reduce to the case when $F=0=F^{\prime}$. Then extensions are classified by elements of $\operatorname{Ext}\left(\left(\mathbb{Q} / \mathbb{Z}_{(p)}\right)^{n}, \mathbb{Z}_{p}^{m}\right) \approx \operatorname{hom}\left(\mathbb{Z}_{p}^{n}, \mathbb{Z}_{p}^{m}\right)$; if $A \in \operatorname{hom}\left(\mathbb{Z}_{p}^{n}, \mathbb{Z}_{p}^{m}\right)$ classifies the extension then

$$
M \approx \operatorname{Cok}\left(\mathbb{Z}_{p}^{n} \stackrel{(A, I)}{\longrightarrow} \mathbb{Z}_{p}^{m} \oplus \mathbb{Q}_{p}^{n}\right)
$$

where $I: \mathbb{Z}_{p}^{n} \rightarrow \mathbb{Q}_{p}^{n}$ is the standard inclusion. It follows that $M \approx \mathbb{Z}_{p}^{m} / \operatorname{im} A \oplus$ $\mathbb{Q}_{p}^{n} /$ ker $A$; this can be shown by choosing a map $B: \mathbb{Z}_{p}^{m} \rightarrow \mathbb{Q}_{p}^{n}$ such that (I$B A): \mathbb{Z}_{p}^{n} \rightarrow \mathbb{Q}_{p}^{n}$ projects to the kernel of $A \otimes \mathbb{Q}$, in which case there is an exact sequence

$$
0 \rightarrow \mathbb{Z}_{p}^{n} \stackrel{(A, I)}{\longrightarrow} \mathbb{Z}_{p}^{m} \oplus \mathbb{Q}_{p}^{n} \stackrel{(x, y) \rightarrow(x, y-B x)}{\longrightarrow} \mathbb{Z}_{p}^{m} / \operatorname{im} A \oplus \mathbb{Q}_{p}^{n} / \operatorname{ker} A \rightarrow 0
$$

which realizes the splitting of $M$. Now the result follows from the fact that $\mathbb{Z}_{p}^{m} / \operatorname{im} A \approx F \oplus \mathbb{Z}_{p}^{a}$ and $\mathbb{Q}_{p}^{n} / \operatorname{ker} A \approx\left(\mathbb{Q} / \mathbb{Z}_{(p)}\right)^{b} \oplus \mathbb{Q}_{p}^{c}$, where $F$ is a finite $p$-group.

8.10. Duality for fp-spectra. Recall that there exist natural transformations $L_{n+1}^{f} X \rightarrow L_{n}^{f} X$, and hence natural transformations $W_{n} X \rightarrow W_{n+1} X$. We define

$$
W X=\underset{n \rightarrow \infty}{\operatorname{hocolim}} W_{n} X
$$

If $X$ is an fp-spectrum with fptype $(X)=n$, then (7.1) shows that $L_{m}^{f} X \approx L_{n}^{f} X$ for $m \geq n$, and thus $W_{m} X \approx W_{n} X$ for $m \geq n$. This, together with (8.3) and the fact that $\mathcal{C}=\bigcup_{n} \mathcal{C}_{n}$ is the homotopy category of all fp-spectra, gives

Theorem 8.11. The functor $W$ induces an equivalence of categories $W: \mathcal{C}^{\mathrm{op}} \rightarrow \mathcal{C}$, and $H_{*} W X \approx \tilde{I}\left(H_{*} X\right)$ for all $X$ in $\mathcal{C}$.

\section{Calculations}

In this section we compute $W X$ in several cases, and thus implicitly compute $L_{n}^{f} X$ (and $L_{n} X$, by (7.2)) for sufficiently large $n$.

Lemma 9.1. If $X$ is a ring spectrum and $Y$ is an $X$-module spectrum, then $W_{n} Y$ is also an $X$-module spectrum.

Proof. This is a formal consequence of the fact that $W_{n} Y \approx \mathcal{F}\left(Y, W_{n} S^{0}\right)$. 
Proposition 9.2. If $X$ is an fp-spectrum which is a ring spectrum, $H_{*} X$ is selfdual as a finitely-presented comodule (i.e., $\tilde{I}\left(H_{*} X\right) \approx \Sigma^{d} H_{*} X$ for some d), and $H_{*} X$ does not split over the Steenrod algebra, then $W X \approx \Sigma^{d} X$.

Proof. Choose a map $S^{d} \rightarrow W X$ which hits the bottom homology class, dual to the unit in $H_{*} X$. By (9.1) this map extends to a map $\Sigma^{d} X \rightarrow W X$ of $X$-module spectra, and this map is necessarily an isomorphism on $\bmod p$ homology, and hence an equivalence.

Corollary 9.3. We have that

1. $W k(n) \approx \Sigma^{2 p^{n}-1} k(n)$,

2. $W B P\langle n\rangle \approx \Sigma^{e(n)} B P\langle n\rangle$, where $e(n)=2 \frac{p^{n+1}-1}{p-1}-(n+1)$,

3. $W b u \approx \Sigma^{4} b u$ (at all primes),

4. $W b o \approx \Sigma^{6}$ bo (at all primes), and

5. $W e o_{2} \approx \Sigma^{23} e o_{2}$ at $p=2$ and at $p=3$.

Proof. The only case which needs comment is 5 . In this case it can be derived from the following facts [5]. At $p=2, e o_{2} \wedge F \approx B P\langle 2\rangle$, where $F$ is a certain finite complex with $H^{*} F \approx \mathcal{D} A^{*}(1)$, the "double" of $A^{*}(1)$. At $p=3, e o_{2} \wedge\left(S^{0} \cup_{\alpha} e^{4} \cup_{2 \alpha}\right.$ $\left.e^{8}\right) \approx B P\langle 2\rangle \vee \Sigma^{8} B P\langle 2\rangle$.

Remark 9.4. In each of the above examples, we can read off $\pi_{*} L_{n} X$ from our knowledge of the homotopy of $X$. In particular, in cases $2-5$ there is a wide "gap" between the first copy of $\mathbb{Z}_{p}$ and the last copy of $\mathbb{Q} / \mathbb{Z}_{(p)}$ in the homotopy of $L_{n} X$; if $W X \approx \Sigma^{d} X$, then

$$
\pi_{s} X \approx \begin{cases}\mathbb{Z}_{p} & \text { if } s=0, \\ 0 & \text { if } 1-d<s<0, \text { and } \\ \mathbb{Q} / \mathbb{Z}_{(p)} & \text { if } s=1-d .\end{cases}
$$

There is a convenient heuristic for reading off the expected size of the "gap" in $\pi_{*} L_{n} X$ for many ring spectra of the above type. If $\pi_{*} X \otimes \mathbb{Q} \approx \mathbb{Q}_{p}\left[x_{1}, \ldots, x_{n}\right]$, then the size of the gap is $\sum_{i=1}^{n}\left(\left|x_{i}\right|+1\right)$. For example, $\pi_{*} e o_{2} \otimes \mathbb{Q} \approx \mathbb{Q}_{p}\left[x_{8}, x_{12}\right]$ at $p=2$ or 3 , so the gap is $(8+1)+(12+1)=22$. For $B P\langle n\rangle$ the gap is the same as the dimension of the Toda complex $V(n)$, should it exist.

Recall from (4.10) that if $H_{*} X \approx A_{*} \otimes_{A_{*}(n)} M$, then $\tilde{I}\left(H_{*} X\right) \approx A_{*} \otimes_{A_{*}(n)} \Sigma^{d} \check{M}$, where $d$ is the dimension of the "top cell" of $A_{*}(n)$.

Proposition 9.5. Let $J_{p}$ denote the connective image-of-J spectrum completed at the prime $p$.

1. For $p$ odd, $W J_{p} \approx \Sigma^{3} J_{p}$.

2. For $p=2$, there is a cofiber sequence $\Sigma^{3} H \mathbb{F}_{2} \rightarrow J_{2} \wedge\left(S^{0} \cup_{2} e^{1} \cup_{\eta} e^{3}\right) \rightarrow W J_{2}$.

Proof. At an odd prime, $J_{p}$ is the fiber of any map $B P\langle 1\rangle \rightarrow \Sigma^{q} B P\langle 1\rangle, q=2(p-1)$, which sends the cohomology generator $\iota \in H^{q} \Sigma^{q} B P\langle 1\rangle$ to $P^{1} \iota \in H^{q} B P\langle 1\rangle$. At $p=2, J_{2}$ is the fiber of any map bo $\rightarrow$ bspin which sends the cohomology generator $\iota_{\text {bspin }} \in H^{4}$ bspin to $S q^{4} \iota_{b o} \in H^{4} b o$ [10]. In either case, the map in cohomology is induced from a map of $A^{*}(2)$-modules.

We leave the odd prime case to the reader. Suppose $p=2$, and let $F=S^{0} \cup_{2}$ $e^{1} \cup_{\eta} e^{3}$. Since bspin $\approx \Sigma^{7} b o \wedge D F$ and $H^{*}(F \wedge D F)$ is a direct sum over the 
Steenrod algebra of a spherical class in dimension 0 with a free $A^{*}(1)$ module on one generator, we see that $J \wedge F$ fits in a fiber sequence

$$
J \wedge F \rightarrow b o \wedge F \rightarrow \Sigma^{7} \text { bo } \vee \Sigma^{4} H \mathbb{F}_{2} .
$$

We can kill the copy of $H \mathbb{F}_{2}$ by taking the evident cofiber $\Sigma^{3} H \mathbb{F}_{2} \rightarrow J \wedge F \rightarrow C$, so that we obtain a cofiber sequence

$$
C \rightarrow b o \wedge F \rightarrow \Sigma^{7} b o
$$

On applying $W$ we get a fiber sequence

$$
\Sigma^{-1} b o \rightarrow \Sigma^{6} b o \wedge D F \rightarrow W C \rightarrow b o \stackrel{f}{\rightarrow} \Sigma^{7} b o \wedge D F .
$$

We can compute the action on $f$ on cohomology, since by (4.10) it is induced from a map of $A^{*}(2)$-modules. The computation, which is straightforward, shows that the bottom class of $\Sigma^{7} b o \wedge D F$ hits $S q^{4} \iota_{b o} \in H^{4} b o$, and thus $W C \approx J$.

Corollary 9.6 (Hopkins).

$$
I\left(L_{1} S^{0}\right) \approx L_{1}\left(S^{-1} \cup_{2} e^{0} \cup_{\eta} e^{2}\right) .
$$

\section{REFERENCES}

[1] A. K. Bousfield. The localization of spectra with respect to homology. Topology, 18:257-281, 1979.

[2] A. K. Bousfield and D. M. Kan. Homotopy Limits, Completions and Localizations. Lecture Notes in Mathematics 304. Springer-Verlag, 1972.

[3] E. H. Brown and M. Comenetz. Pontrjagin duality for generalized homology and cohomology theories. Amer. J. Math., 98:1-27, 1976.

[4] M. J. Hopkins and B. H. Gross. The rigid analytic period mapping, Lubin-Tate space, and stable homotopy theory. Bulletin of the Amer. Math. Soc., 30:76-86, 1994.

[5] M. J. Hopkins and M. Mahowald. On the spectrum $e o_{2}$. in preperation.

[6] M. J. Hopkins and J. H. Smith. Nilpotence and stable homotopy theory, II. to appear in Ann. of Math.

[7] M. Hovey, J. H. Palmieri, and N. P. Strickland. Axiomatic Stable Homotopy Theory. Number 610 in Memoirs of the Amer. Math. Soc. Amer. Math. Soc., 1997.

[8] M. Hovey and N. P. Strickland. Morava $K$-theories and localisation. to appear in Memoirs of the Amer. Math. Soc.

[9] M. Mahowald. bo-resolutions. Pacific J. Math., 92:365-383, 1981.

[10] M. Mahowald and R. J. Milgram. Operations which detect $S q^{4}$ in connective $K$-theory and their applications. Quart. J. Math. Oxford (2), 27:415-432, 1976.

[11] M. Mahowald and H. Sadofsky. $v_{n}$-telescopes and the Adams spectral sequence. Duke Math. J., 78:101-129, 1995.

[12] H. R. Margolis. Spectra and the Steenrod Algebra. North-Holland, 1983.

[13] S. A. Mitchell. Finite complexes with $A(n)$-free cohomology. Topology, 24:227-248, 1985.

[14] D. C. Ravenel. Localization with respect to certain periodic homology theories. Amer. J. Math, 106 (1):351-414, 1984.

[15] D. C. Ravenel. Complex Cobordism and Stable Homotopy Groups of Spheres. Academic Press, 1986.

[16] D. C. Ravenel. Nilpotence and Periodicity in Stable Homotopy Theory. Annals of Mathematics Studies. Princeton University Press, 1992.

[17] H. Sadofsky. Hopkins' and Mahowald's picture of Shimomura's $v_{1}$-Bockstein spectral sequence calculation. In M. Tangora, editor, Algebraic Topology (Oaxtepec, 1991), number 146 in Contemp. Math., pages 407-418. Amer. Math. Soc., 1993.

[18] K. Shimomura. On the Adams-Novikov spectral sequence and products of $\beta$-elements. $\mathrm{Hi}$ roshima Math. J., 16:209-224, 1986.

[19] K. Shimomura and A. Yabe. The homotopy groups $\pi_{*}\left(L_{2} S^{0}\right)$. Topology, 34:261-290, 1995. 
Department of Mathematics, Northwestern University, Evanston, IL 60208

E-mail address: mark@math.nwu.edu

E-mail address: rezk@math.nwu.edu 